\title{
L'orario del pasto altera la tolleranza glicidica, l'ossidazione del substrato e le variabili circadiano-correlate: uno studio randomizzato incrociato
}

\author{
Paola Fierabracci ${ }^{1}$
}

Pubblicato online: 14 luglio 2015

(C) Springer International Publishing AG 2015

\section{Commento a:}

Meal timing affects glucose tolerance, substrate oxidation and circadian-related variables:

a randomized, crossover trial.

C. Bandín, F.A. Scheer, A.J. Luque, V. Ávila-Gandía, S. Zamora, J.A. Madrid, P. Gómez-Abellán, M. Garaulet.

Int J Obesity (2015) 39:828-833

Studi precedenti hanno dimostrato che l'orario in cui avviene l'assunzione del pasto influenza la regolazione del peso corporeo, la sensibilità insulinica e la tolleranza glucidica con meccanismi non noti; comunque questi effetti sembrano indipendenti dall'introito calorico nelle 24 ore e dai livelli di attività fisica. Lo scopo dello studio era quello di valutare gli effetti dell'orario del pasto sul consumo energetico a riposo, sulla termogenesi indotta dalla dieta, sull'ossidazione del substrato, sulla tolleranza glucidica e sulle variabili correlate al sistema circadiano quali la variazione del cortisolo e della temperatura del polso come espressione dell'effetto del pasto sul metabolismo basale.

Sono stati esaminati due gruppi di giovani donne sane, normopeso, alle quali veniva somministrato un pasto standard in due orari diversi del pranzo, precoce alle 13 (EE) e tardivo (LE) alle 16,30 per 2 settimane per ciascun protocollo.

LE rispetto a EE determinava una riduzione del consumo energetico a riposo misurato con la calorimetria indiretta nella fase pre-prandiale, una ridotta tolleranza glucidica, una riduzione della concentrazione del cortisolo salivare del mattino e del pomeriggio e una riduzione della temperatura del polso dopo il pasto.

In studi precedenti condotti su un numero elevato di soggetti obesi gli autori avevano osservato che l'orario del pasto principale era predittivo dell'entità del calo ponderale e che la sensibilità insulinica era minore in chi assumeva il pasto in una fase tardiva. In questo articolo Bandin e collaboratori dimostrano che anche in soggetti sani e non obesi l'orario del pasto può modulare il metabolismo basale. Anche se lo studio è stato condotto in un piccolo campione di soggetti e per un tempo limitato, può costituire una base fisiologica per comprendere meglio la neuromodulazione del metabolismo energetico; inoltre queste osservazioni, da un punto di vista pratico, possono essere utilizzate per favorire il calo ponderale in un programma dietetico comportamentale nel trattamento a lungo termine dell'obesità.

\footnotetext{
P. Fierabracci

pfierab2001@yahoo.it

1 UO Endocrinologia I, Azienda Ospedaliero Universitaria Pisana, Pisa, Italia
} 